Nutzungsintensivierung und Nutzungsdauerverlängerung - ein kleines Fazit

\title{
Mehr Ökonomie als Ökologie
}

\author{
Nutzungsintensivierung und Nutzungsdauerverlängerung sind ein wichtiges \\ Thema im ökologischen Diskurs. Sie gelten als vielversprechende Methoden, \\ den Umfang von Stoffströmen und damit die Belastung der Umwelt zu reduzie- \\ ren. Die folgenden sieben Thesen würdigen ihre ökonomische und ökologische \\ Bedeutung; sie warnen jedoch auch vor übertriebenen Erwartungen.
}

$\mathrm{T}$ hese 1: Nutzungsintensivierung und Nutzungsdauerverlängerung müssen sich rechnen.

Nutzungsintensivierung ist zumeist mit einer Übertragung von Verfügungsrechten auf Dritte verbunden. Diese Übertragung verursacht in der Regel Transaktionskosten. Sie lohnt sich deshalb nur dann, wenn die erzielbaren Kostenreduktionen (im weitesten Sinne des Wortes) im Rahmen eines solchen neuen institutionellen Arrangements oder ein möglicher Nutzenzuwachs die anfallenden Transaktionskosten übersteigen.

Bei Strategien der Nutzungsdauerverlängerung ist das Verhältnis zwischen den Reparaturkosten einerseits und den Anschaffungskosten andererseits die entscheidende Größe. Sind die Reparaturkosten relativ hoch oder ist die Diskrepanz zwischen Reparaturkosten und Anschaffungskosten gering, dann besteht nur ein geringer Anreiz Geräte zu reparieren. Umgekehrt ist der Anreiz zur Neuanschaffung dann entsprechend größer.

Diese an und für sich banalen Aussagen bekommen ein wenig Farbe, wenn man sie in einen $\mathrm{Zu}$ sammenhang mit der Diskussion um den ökologischen Konsum stellt. Sie besagen dann, dass ökologische Motive letztlich nicht die entscheidenden Motive sind, die Konsumenten dazu veranlassen könnten, Nutzungsintensivierung oder Nutzungsdauerverlängerung zu betreiben. Ausnahmen bestätigen natürlich die Regel, insbesondere dann, wenn es um die Entstehung von Marktnischen geht, in denen solche Strategien eine Lernkurve durchlaufen, die Produktivität und die Logistik verbessert werden und damit das PreisLeistungs-Verhältnis gegenüber anderen Nutzungsformen verbessert wird. Aber unter dem Strich gilt eben: auch Nutzungsintensivierung und Nutzungsdauerverlängerung müssen sich erst einmal rechnen.

\section{- Pfadabhängigkeit des Konsums}

These 2: Strategien zur Nutzungsintensivierung und Nutzungsdauerverlängerung sind pfadabbängig.

In der Regel werden Konsumentscheidungen kaum oder gar nicht rational kalkuliert; vorherrschend ist die habituelle Entscheidung. Das hat gute Gründe: Würde jede Konsumentscheidung Gegenstand eines rationalen Kalküls mit einer entsprechend gründlichen umfangreichen Informationsbeschaffung, dann wären wir vor lauter Entscheidungsproblemen kaum noch in der Lage, tatsächlich Entscheidungen zu fällen.

Dieser Mechanismus sorgt dafür, dass alte Konsumentscheidungen zunächst einmal tradiert werden, solange keine gravierenden Veränderungen der Umfeldbedingungen dieser Entscheidung oder der Präferenzen der Entscheider stattgefunden haben. Die Konsumentscheidungen sind mithin pfadabhängig.

$\mathrm{Zu}$ den sozialen Mechanismen, die eine solche Pfadabhängigkeit begünstigen, zählen auch der Wunsch, einen bestimmten Lebensstil oder eine bestimmte personelle Identität durch ähnliche Konsumentscheidungen aufrecht zu erhalten, und die Imitation von Konsumentscheidungen von sozialen Bezugsgruppen, die als attraktiv empfunden werden. Deshalb verwundert es nicht, dass zukünftige Wahlentscheidungen über Konsumalternativen immer noch am besten durch den Rückgriff auf vergangene Konsumentscheidungen in ähnlich gelagerten Situationen prognostiziert werden können. Eine ähnliche tradierende Wirkung auf Konsumentscheidungen haben die Infrastrukturen technischer Art, in die diese Konsumentscheidungen eingebettet sind. Sie sorgen ebenfalls dafür, dass bestimmte Entscheidungen immer wieder gegenüber anderen Alternativen vorgezogen werden, einfach deshalb, weil sie infrastrukturell begünstigt sind.
Von besonderem Interesse sind daher natürlich Umbruchsituationen. Das sind Situationen, in denen neue Konsumentscheidungen, auch solche für Nutzungsintensivierung oder Nutzungsdauerverlängerung, eine Chance haben. Solche Umbrüche können dadurch ausgelöst und begünstigt werden, dass

- sich der infrastrukturelle Rahmen durch Innovationen ändert,

sich die fraglichen Preis-Leistungs-Verhältnisse konkurrierender Konsumalternativen durch Produktivitätsfortschritte verändern oder dass

Veränderungen der kognitiven Strukturen und Werte der Beteiligten statt finden.

Vor allem in solchen Situationen herrschen günstige Voraussetzungen für Verhaltensinnovationen, also Änderungen der Konsumentscheidungen. Diese können, müssen aber natürlich nicht zugunsten von Nutzungsintensivierung und Nutzungsdauerverlängerung genutzt werden.

\section{Weit verbreitete Strategien}

These 3: Nutzungsintensivierung und Nutzungsdauerverlängerung sind völlig normale und weit verbreitete Bestandteile alltäglichen Verhaltens in modernen Gesellschaften.

Traditionell dominiert im ökologischen Diskurs die Vorstellung, dass Nutzungsintensivierung und Nutzungsdauerverlängerung Nischenstrategien seien, für deren Verbreitung ein großer Aufwand getrieben werden muss. Das ist zutreffend, wenn der Blickwinkel auf solche Strategien beschränkt wird, die explizit ökologisch motiviert sind. Wenn wir uns aber den Gedanken erlauben, dass Nutzungsintensivierung und Nutzungsdauerverlängerung auch völlig ohne ökologische Motive statt finden können, dann kann man schnell feststellen, dass wir in unserem gesamten Alltag von Nutzungsintensivierung umgeben sind.

Einige Beispiele können das illustrieren:

- Nutzungskaskaden, die typisch sind für Babywäsche und Kleinkindbekleidung,

die Kurzfristvermietung von Wohnungen und Häusern, die anderenfalls während der Urlaubszeit unbeaufsichtigt wären, oder

der umfangreiche Gebrauchtwagenhandel und das Wiederaufrüsten von gebrauchten Fahrzeugen, mit dessen Hilfe insbesondere einkommensschwächere Schichten die Möglichkeit erhalten am motorisierten Individualverkehr teilzunehmen.

Die Liste der Beispiele ließe sich endlos verlängern. Gemeinsam ist allen solchen Beispielen, dass entweder handfeste ökonomische Motive, die Erweiterung von Nutzungsmöglichkeiten oder aber besondere 
Bequemlichkeits- und Komfortaspekte die Konsumenten motivieren, Nutzungsintensivierung oder Nutzungsdauerverlängerung zu betreiben.

Ein Rückblick in die Geschichte zeigt überdies, dass ursprünglich einmal alle Produktionsaktivitäten Aktivitäten waren, die innerhalb eines Haushaltes abgewickelt wurden. Die Übertragung von Verfügungsrechten über solche Produktionsaktivitäten auf Dritte können wir einerseits als Entstehung der modernen arbeitsteiligen Gesellschaft begreifen; sie ist andererseits aber auch gleichbedeutend mit jenem Outsourcingprozess, der typisch ist für Strategien der Nutzungsintensivierung. Insofern ist diese organisatorische Form ein völlig normaler Bestandteil der historischen Genese des Kapitalismus.

Nicht so klar ist das Bild bei der Nutzungsdauerverlängerung. Die Geschichte der Kapitalismuskritik ist voll von Vorwürfen über den eingebauten Verschleiß von Produkten. Insbesondere dann, wenn der Wettbewerb sehr intensiv ist, muss man offensichtlich damit rechnen, dass die Hersteller kein sonderliches Interesse an der Langlebigkeit ihrer Produkte haben. Das lässt sich gut am Vergleich zwischen der deutschen und der US-amerikanischen Produktkultur demonstrieren.

Soviel ist immerhin klar: In einer Welt, die sich durch ständige technische und modische Neuerungen auszeichnet, scheint es oft nicht sinnvoll zu sein, den Produkten ein Höchstmaß an technischer Lebensdauer mitzugeben. Ein Plattenspieler mit einer Lebensdauer von 100 Jahren ist in einer Welt, in der die Speichermedien für Musik ständig wechseln, kein verkaufbares Produkt. Umgekehrt gilt aber auch, dass die technischen Möglichkeiten, Produkte langlebiger zu machen, erheblich zugenommen haben, nicht zuletzt durch die in der Literatur oft bemühte Modulbauweise.

\section{Ökologische Motive sind selten}

These 4: Ökologischer Konsum ist eher die Ausnahme.

Zwischen Entscheidungen und Handlungen sowie den Folgen, die diese Entscheidungen und Handlungen auf Dritte haben besteht eine Diskrepanz. Diese ist in unserer Gesellschaft gerade mit Blick auf ökologische Probleme so groß, dass es für den Einzelnen nur selten möglich ist, eine individuelle Zurechnung von Folgen zu Entscheidungen vorzunehmen. Vor diesem Hintergrund sind Kaufentscheidungen, die die Natur schonen, eine Ausnahme. Zweitens wirkt eine Einstellung, die in der angelsächsischen Literatur Consumerism genannt wird, also jene Spirale von Einkommenssteigerung durch Arbeit und Erweiterung der Konsummöglichkeiten als Belohnung für die Arbeit, begünstigend auf eine Ausweitung des materiellen Konsums. Diese Einstellung, die das zentrale Glücksversprechen moderner Gesellschaften transportiert, ist tief in die Mentalität der Industriekulturen eingegraben, und nicht so ohne weiteres zu ändern. Schließlich gibt es drittens eine Verknüpfung zwischen Status und dem Besitz an bestimmten langlebigen materiellen Konsumgütern, die ebenfalls ökologischen Konsum aus ethischen Motiven erschwert.

Ökologischer Konsum im Sinne einer mit ökologischen Motiven getroffenen Konsumentscheidung ist also eine Ausnahme von der Regel. Er ist aber eine wichtige Ausnahme, weil ökologische Konsumentscheidungen Pionierentscheidungen sein können, die andere Konsummuster vorstellen und damit nachahmungsfähig machen. Beispiele sind etwa die Verbreitung von Müsli oder von Eiern von freilaufenden Hühnern in den Supermarktregalen, von Produkten also, deren Verbreitung von ökolo-

\section{punkt-um.net}

Ihre Internet-Plattform zum Infodienst punkt.um

für Informationen zu Nachhaltigkeit und Ökologie

Aktuelle Termine - News aus dem Internet - Website-Datenbank

Ökomedia: Abstracts-Datenbank zu 70.000 Zeitschriftenartikeln und Bücher seit 1987

Sie finden uns unter: http://www.punkt-um.net

gisch motivierten Pionieren betrieben wurde und die heute bereits am Frühstïcksbuffet eines Mittelklassehotels zu finden sind.

\section{Hemmnis Statuskonsum}

These 5: Statuskonsum und Eigentumsbindung sind Hindernisse für Nutzungsintensivierung und Nutzungsdauerverlängerung.

Das Streben nach Status ist in gewisser Weise eine Konstante menschlicher Gemeinschaften. Status ist in modernen Industriegesellschaft an den Besitz materieller Güter gebunden. Soziale Subgruppen und historische Betrachtungen zeigen allerdings, dass das nicht zwangsläufig so sein muss. Status muss sich nicht über den Besitz eines leistungsstarken Autos oder eines großen Hauses vermitteln, er kann auch an Bildung oder an bestimmte Fähigkeiten gekoppelt sein.

Selbst wenn man also annimmt, dass das Differenzierungsverlangen über Status eine Art anthropologische Konstante darstellt, folgt daraus nicht zwangsläufig, dass sich jedermann mit einem bunten Blumenstrauß materieller Güter umgibt. Die Erwartung, dass in der Informationsgesellschaft die klassische Trias von Auto, Eigenheim und Fernreise an Bedeutung für den Status verliert und andere Statussymbole wie Computer oder Auslandsaufenthalt an Bedeutung gewinnen, ist nicht von der Hand zu weisen. Solche Veränderungen der Gegenstände oder Fähigkeiten, die den Status ausmachen, sind langwierige kulturelle Prozesse und lassen sich kaum politisch steuern. Es wäre wohl auch nicht wünschenswert, wenn man es versuchen würde.

Die Eigentumsorientierung vermittelt eine gewisse Sicherheit in einer arbeitsteiligen Gesellschaft, weil sie zumindest den Eindruck vermittelt, dass die Dinge, die einem gehören, damit auch zur eigenen Verfügung stehen. Darüber hinaus gilt für viele materielle Dinge, dass sie symbolischen Konsum repräsentieren: mit den materiellen Dingen, mit denen wir uns umgeben, zeigen wir, wer wir sind oder zumindest wer wir sein wollen. Die durch die Eigentumsbindung vermittelte Sicherheit ist allerdings in hohem Maße eine Illusion, denn der Besitz eines Hauses wäre wenig wert, wenn die Infrastruktursysteme wie Wasser, Abwasser, Energie und Telefon nicht funktionieren würden, und ein Auto ohne ein ausgebautes Straßensystem und eine gesicherte Energiezufuhr ist auch kaum zu gebrauchen. Kurzum: wir sind bei der Nutzung aller unserer kostbaren Eigentiimer auf das reibungslose Funktionieren einer arbeitsteiligen Gesellschaft angewiesen. Individuelle Verfügungsrechte sind da lediglich not- 
wendige, aber wohl fast nie hinreichende Bedingung für die Sicherung von Konsummöglichkeiten in einer modernen Gesellschaft.

\section{- Hilfreiche Trends}

These 6: Es gibt einige Trends, die die weitere Verbreitung von Strategien der Nutzungsintensivierung und Nutzungsdauerverlängerung unterstützen.

Als solche Trends sind unter anderem zu nennen:

- Nutzungsintensivierung und Nutzungsdauerverlängerung sind zwei prominente Diversifizierungsstrategien in gesättigten oder stagnierenden Märkten.

- Durch Fortschritte auf dem Gebiet der Informationstechnik und der Logistik sinken die Transaktionskosten. Das begünstigt Outsourcingprozesse, die Nutzungsintensivierung oft begleiten.

- Es findet eine Senkung der Haushaltsproduktivität durch die demographische Entwicklung statt. Es gibt immer mehr Einpersonenhaushalte durch die Trends zur Versingelung und zur zunehmenden Überalterung der Gesellschaft. Damit werden Dienstleistungskonzepte zunehmend attraktiv.

- Eigentumsbindungen an materielle Güter sind ein Ballast, wenn von den Individuen in der mo- dernen Gesellschaft in zunehmendem Maße räumliche Mobilität und zeitliche Flexibilität gefordert werden.

- Der Trend zur Informationsgesellschaft entwertet den Zusammenhang zwischen Status und bestimmten langlebigen Konsumgütern. Zunehmend bedeutsam für den sozialen Status werden bestimmte Fähigkeiten im Umgang mit Informationen und Informationsverarbeitungsmaschinen.

\section{Ambivalenter Umwelteffekt}

These 7: Der ökologische Ertrag von Nutzungsintensivierung und Nutzungsdauerverlängerung ist ambivalent.

Wenn die These 1 , wonach sich à la longue Nutzungsintensivierung und Nutzungsdauerverlängerung rechnen müssen, richtig ist, dann führen solche Strategien zu einer Kostensenkung für den Verbraucher. Mit anderen Worten: er kann das gleiche Nutzenniveau mit einem geringeren Einkommen realisieren. Das bedeutet, dass Einkommen frei und zusätzliche Kaufkraft geschaffen wird. Im Prinzip kann diese indirekte Einkommenssteigerung auf zwei Arten und Weisen genutzt werden: Entweder man kann bei gleichbleibendem Konsumniveau weniger arbeiten oder aber man kann den Konsum ausweiten. Die vergangenen 50 Jahre haben gezeigt, dass die Produktivitätssteigerung der Arbeit - etwa um den Faktor Vier - fast ausschließlich zugunsten der Erweiterung der Konsummöglichkeiten genutzt wurde und nicht zu einer Reduktion der Lebensarbeitszeit. Es ist nicht so recht zu sehen, dass dies bei dem beschriebenen Einkommenseffekt anders sein sollte.

Das muss natürlich in Zukunft nicht so bleiben, vermittelt aber dennoch eine gewisse Skepsis mit Blick auf den ökologischen Ertrag einer Ressourcenproduktivitätssteigerung durch Nutzungsintensivierung und Nutzungsdauerverlängerung, hängt er doch davon ab, welche neuen Konsummöglichkeiten sich die Konsumenten mit ihrer frei werdenden Kaufkraft erschließen.

\section{Der Autor}

Dr. Stefan Zundel ist Professor für Öffentliche Finanzwirtschaft, Energie und Umwelt an der Fachhochschule Lausitz. Er war drei Jahre lang Berater des vom IÖW durchgeführten BMBF-Projekts "Neue Nutzungskonzepte für Produkte".

Kontakt: FH Lausitz, Großenhainer Straße 57, 01968 Senftenberg, Tel. 03573/85-733, Fax -709, E-mail: zundel@ww.fh-lausitz.de

\section{Literatur zum Schwerpunkt}

- Bagschik, Thorsten: Gebrauchsüberlassung komplexer Konsumgüter, Wiesbaden 1999.

- Behrendt, Siegried/ Pfitzner, Ralf: Eco-Services for Sustainable Development in the European Union. Renting, Leasing, Sharing and Pooling Services - Methodology and State of the Art, IZT WerkstattBericht Nr. 33, Berlin 1999.

- Behrendt, Siegfried/ Pfitzner, Ralf/ Kreibich, Rolf: Wettbewerbsvorteile durch ökologische Dienstleistungen. Umsetzung in der Unternehmenspraxis, Berlin et al. 1999.

- Berndt, Arnold: Die Erhöhung von Ressourcenproduktivität durch die gemeinsame Nutzung von Gütern. Eine ökonomische Analyse, Wuppertal Papers Nr. 82, Wuppertal 1998.

- Bierter, Willy: Ökologisches Produkt-Design, öko-intelligente Dienstleistungs- und Nutzungskonzepte im Bereich Heimmöbel, Giebenach/Genf 1999.

- Bierter, Willy/Stahel, Walter/Schmidt-Bleek, Friedrich: Öko-intelligente Produkte,

Dienstleistungen und Arbeit, Wuppertal Spezial 2, Wuppertal 1996

- Canzler, Weert/ Franke, Sassa: Autofahren zwischen Alltagsnutzung und Routinebruch, Forschungsbericht des WZB FS II 00-102, Berlin 2000.
- Deutsch, Christian: Abschied vom Wegwerfprinzip. Die Wende zur Langlebigkeit in der industriellen Produktion, Stuttgart 1994.

- Empacher, Claudia: Öko-Dienstleistungen. Begriff und Bedeutung, Materialien Soziale Ökologie 5, Frankfurt/ M. 1994.

- Franck, Egon/ Bagschik, Thorsten/ Opitz, Christian/ Pudack, Torsten: Strategien der Kreislaufwirtschaft und mikroökonomisches Kalkül, Stuttgart 1999.

- Hirschl, Bernd: Produkte länger und intensiver nutzen - das Beispiel private Textilwäsche, Schriftenreihe des IÖW Nr. 149/00, Berlin 2000.

- Hockerts, Kai: Konzeptualisierung ökologischer Dienstleistungen, IWÖ Diskussionspapier Nr. 29, St. Gallen 1995.

- Hrauda, G./ Jasch, C.: Eco-services. Bericht Österreich, Wien 1998.

- Hübner, M. et al.: Produkte für Dienstleistungsanbieter, Wien 2000.

- Klemmer, Paul/ Hinterberger, Fritz (Hrsg.): Ökoeffiziente Dienstleistungen, Wuppertal 1999.

- Konrad, Wilfried: Produkte länger und intensiver nutzen - das Beispiel Wintersport, Schriftenreihe des IÖW Nr. 148/00, Berlin 2000.
- Leinkauf, Simone/ Zundel, Stefan: Funktionsorientierung und Ökoleasing. Strategien und Instrumente einer proaktiven Umweltpolitik, Schriftenreihe des IÖW Nr. 79/94, Berlin 1994.

- OECD (Ed.): Product Durability and Product Life Extension, Paris 1982.

- Scholl, Gerd Ulrich/ Hirschl, Bernd/ Tibitanzl, Frank: Produkte länger und intensiver nutzen. Zur Systematisierung und ökologischen Beurteilung alternativer Nutzungskonzepte, IÖW Schriftenreihe 134/98, Berlin 1998.

- Schrader, Ulf: Empirische Einsichten in die Konsumentenakzeptanz öko-effizienter Dienstleistungen, Lehr- und Forschungsbericht Nr. 42 des Lehrstuhl Markt und Konsum, Hannover 1998.

- Stahel, Walter: Vertiefungsstudie zu Langlebigkeit und Materialrecycling im Bereich der Produkte, Stuttgart 1991.

- Stahel, Walter: Allgemeine Kreislauf- und Rückstandswirtschaft. Intelligente Produktionsweisen und Nutzungskonzepte, Karlsruhe 1996.

- Swedish Environmental Protection Agency (Ed.): Product-Service Systems. AFR-report 288, Lund 2000.

- Verbraucherzentrale Baden-Württemberg (Hrsg.): Nutzen statt Besitzen. Mieten, Teilen, Leihen von Gütern. Ein Zukunftsmodell?, Stuttgart 1996. 
(c) 20I0 Authors; licensee IÖW and oekom verlag. This is an article distributed under the terms of the Creative Commons Attribution Non-Commercial No Derivates License (http://creativecommons.org/licenses/by-nc-nd/3.o/), which permits unrestricted use, distribution, and reproduction in any medium, provided the original work is properly cited. 